\title{
Effects of Nutrition Health Education and Targeted Nutrition Guidance on Maternal Nutritional Status and Maternal and Infant outcomes
}

\author{
Shuli Yang ${ }^{1}$, Lihui Si, Yan Jia, Wenwen Jian, Qing Yu, Min Wang, Ruixin Lin ${ }^{\text {* }}$ \\ ${ }^{1}$ The Second Hospital of Jilin University, Changchun city Jilin Province, 130012 \\ axddfc@126.com
}

Keywords: Nutrition health education, Targeted nutrition guidance, Nutritional status

\begin{abstract}
Objective: To explore the effects of nutritional health education and targeted nutrition guidance on maternal nutritional status and maternal and infant outcomes. Methods: Select 78 Cases of pregnant women received by our hospital in February 2017-December as the objects of this study, randomly divide them into two groups. With the consent of patients, we carry out conventional nursing model on one group and adopt nutrition health education and target nutrition guidance intervention on another group. Then, we compare the complication control effect, outcome of pregnancy and the mode of delivery. Results: Compared with the control group, the nutritional group has an obvious decrease in terms of diabetes incidence of hypertension, macrosomia and fetal distress and there is a significant difference $(\mathrm{p}<0.05)$; in addition, the the incidence rate of anemia after childbirth is lower compared to control group and the percentage of natural birth significantly increased with a a significant difference $(p<0.05)$. Conclusion: Nutrition health education and targeted nutrition guidance can significantly reduce the complications of pregnant women, improve the nutritional quality of patients, improve the percentage of natural birth. All those effects are obvious and worthy of clinical application.

The fetal stage is a special period of life, and it is also the most basic period. The fetus grows and develops in the mother's uterus. At each stage of its development, the fetus absorbs its nutrients and excretes metabolites. Because the growth and development of the fetus depend mainly on the mother, therefore, the maternal nutritional status directly affects the development of the fetus. Nutritional status has a direct impact on fetal health and can also affect subsequent physical health. Many pregnant women do not have adequate scientific nutrition knowledge and good eating habits, leading to irrational dietary structures and low nutritional levels, which not only affect their physical health but also affect the health of fetus. Therefore, a comprehensive understanding of the dietary supplements and nutritional status of pregnant women along with targeted nutrition education, popularization of nutritional knowledge, improvement of diet structure, correctness of eating habits will prevent nutritional deficiencies, improve physical fitness, and ensure fetal health with prominent and practical significance ${ }^{[1-2]}$. Therefore, a reasonable and balanced intake of nutrients is not only critical for their healthy growth, but also a reasonable understanding of the future work. Therefore, to strengthen learning and continuously improving nutritional knowledge will effectively improve the health of pregnant women. Nutrition education aims to improve people's nutritional status and prevent diseases by improving people's nutritional knowledge and dietary habits and attitudes. Nutrition education is a targeted, planned, and organized education activity. It aims to help and encourage pregnant women to establish a sense of nutrition enhancement, disseminate a certain amount of nutrition knowledge, and encourage people to voluntarily take a diet that is conducive to nutrition, formulate a healthy living habits so as to improve health and life, which can be considered as a convenient and economical intervention ${ }^{[3-4]}$. Nutrition education is an important method to improve nutritional status, which is also an important means to guide scientific and reasonable choice of reasonable meals and establish a healthy lifestyle. Therefore, this article analyzes the effects on maternal nutritional status and maternal and infant outcomes based on nutrition and health education as well as targeted nutritional interventions.
\end{abstract}




\section{Materials and Methods}

\section{General Information}

We have 78 pregnant women undergoing regular pregnancy tests in our hospital based on clinical data. They are randomly divided into a control group and a nutrition group. There are 39 women in the nutrition group, age around 22-37, average age around (25.5 \pm 5.3$)$; the control group has 39 pregnant women, age around $20-36$, average age around $(24.6 \pm 3.7)$. All pregnant women are approved by the Ethical Committee of the Hospital and signed a consent form. By comparing the age, gestational age and heart rate between the two groups, we found out that the $P$ value is greater than 0.05 and is not statistically significant but is comparable. Exclusion criteria: pregnant women with severe medical conditions, mental disorders, can not successfully exchange; combined liver and kidney failure, severe cardiovascular and cerebrovascular disease. The inclusion criteria include single-pregnant women; good compliance, and no other serious complications. There is no statistically significant difference in gender, age, clinical performance, and general data between the two groups $(\mathrm{P}>0.05)$, and they are comparable.

\section{Methods ${ }^{[5-7]}$}

The pregnant women in the control group use the hospital's regular education model. The nutrition group adopts nutrition and health education as well as targeted nutritional interventions. The nutrition and health education mainly promotes regular health education based on multimedia while professional nutritionists can also give classroom explanation and personal demonstrations, give them nutrition-related health knowledge manuals, encourage family members to learn nutrition knowledge together so as to ask family members to supervise on the nutrition of pregnant women. Patients are given continuous inspection so as to monitor the development of fetus with detailed records. In addition, while promoting nutrition knowledge, professionals should be gentle and patient to pass on the knowledge about nutrition and dietary pagoda, let them know the relation between the pregnancy nutrition status and pregnancy outcomes, focus on their pregnancy nutrition so as to promote maternal active cooperation. What's more, pregnancy complications as well as physiological changes should be told to them with nutrition management measures. For example, we should answer various nutrition questions, give lectures to let them know the importance of maternal nutrition, common food nutrient content, energy and nutrition during pregnancy needs, good food match etc. Furthermore, it is necessary to make repeated counseling with the patient or carry out friendly and kind communication, increase the confidence of pregnant women and establish their own confidence.

Targeted nutrition guidance: set up a special nutrition management department to establish pregnancy care card for pregnant women, record the results, body and weight condition along with targeted nutrition guidance based on actual situation. Dietary guidelines: Guide patients to establish a clear and reasonable diet, pregnant women must control the diet and tailor to different individual diet plan. It is best to recommend recipes based on their food preferences so as to clear food type and quantity. In every two weeks, we should give telephone guidance to them along with reasonable dietary advice, encourage them to do outdoor activities and appropriate exercise such as walking after dinner, yoga etc. The accompany of family members or sun is more conducive to natural childbirth.

\section{Evaluation Indicators}

Questionnaire surveys are conducted on two groups and related cases are reviewed for general information regarding the names, ages, heights, pre-pregnancy weights, maternal history, hemoglobin in early pregnancy, and past medical history. The body mass growth during pregnancy, the incidence of complications (gestational diabetes, hypertension, macrosomia), the mode of childbirth outcomes (natural delivery, cesarean section), etc. are analyzed.

\section{Statistical Methods}

All data in this study are processed using SPSS 17.0 statistical software. Measured data are expressed as mean \pm standard deviation $( \pm \mathrm{s}), \mathrm{t}$ is used for test; count data is expressed as adoption rate $(\%)$ and chi-squre is used for test, if $\mathrm{P}<0.05$, it shows difference along with statistical significance. 


\section{Result}

\section{Complications during Pregnancy for Two Groups}

Based on the results, compared with the control group, the incidence of gestational diabetes, gestational hypertension, macrosomia and fetal distress of the nutrition group demonstrate a declining trend in the pregnant period, and the difference is significant $(\mathrm{P}<0.05)$.

Table 1. Complications during pregnancy for two groups

\begin{tabular}{ccccccc}
\hline Group & $\mathrm{n}$ & $\begin{array}{c}\text { Gestational } \\
\text { diabetes }\end{array}$ & $\begin{array}{c}\text { Gestational } \\
\text { hypertensio } \\
\mathrm{n}\end{array}$ & $\begin{array}{c}\text { Macrosom } \\
\text { ia }\end{array}$ & $\begin{array}{c}\text { Fetal } \\
\text { distress }\end{array}$ & $\begin{array}{c}\text { Anemi } \\
\mathrm{a}\end{array}$ \\
$\begin{array}{c}\text { Nutrition } \\
\text { group }\end{array}$ & 39 & 1 & 1 & 2 & 0 & 9 \\
$\begin{array}{c}\text { Control group } \\
\mathrm{P}\end{array}$ & 39 & 6 & 8 & 5 & 4 & 25 \\
\hline
\end{tabular}

\section{Birth Delivery Outcomes of Two Groups}

The rate of cesarean section is up to $51.3 \%$ in the control group, the rate of cesarean section in the nutrition group is only $6.2 \%$. We compare the rate of cesarean section between the two groups and the difference was significant $(\mathrm{p}<0.05)$. The results showed that the cesarean section of nutrition group decrease significantly, as shown in table 2 .

Table 2. Birth delivery outcomes of two groups

\begin{tabular}{cccc}
\hline Group & $\mathrm{n}$ & $\begin{array}{c}\text { Cesarean section } \\
(\%)\end{array}$ & Natural birth (\%) \\
\hline Nutrition group & 39 & $13(33.3)$ & $26(66.7)$ \\
Control group & 39 & $20(51.3)$ & $19(48.7)$ \\
\hline
\end{tabular}

\section{Newborn Babies' Weight}

The weight of the newborns in the two groups is recorded. The abnormal rate of neonatal body weight in the control group is $23.1 \%$, and the abnormal rate of neonatal body weight in the nutrition group is $5.1 \%$, which is $18 \%$ lower than that in the control group. There is significant difference $(\mathrm{P}$ $<0.05$ ), but no neonatal death occurred in either group. See Table 3.

Table 3. Newborn babies' weight condition

\begin{tabular}{cccc}
\hline Group & $\mathrm{n}$ & $\begin{array}{c}\text { Normal body weight } \\
(\%)\end{array}$ & $\begin{array}{c}\text { Abnormal body weight } \\
(\%)\end{array}$ \\
\hline $\begin{array}{c}\text { Nutrition } \\
\text { group }\end{array}$ & 39 & $37(94.9 \%)$ & $2(5.1 \%)$ \\
Control group & 39 & $30(76.9 \%)$ & $9(23.1)$ \\
\hline
\end{tabular}

\section{Discussion}

At present, due to different levels of cognition in life, pregnant women suffer from malnutrition or excess in varying degrees during the important period of pregnancy, causing a series of problems that should not exist. Therefore, in the perinatal care, we should pay more attention to nutrition counseling and guidance, so that pregnant women must be aware of the importance of nutrition and dietary balance during pregnancy to ensure the health of pregnant women and the normal development of the fetus. With the great changes in people's lifestyles recently, pregnant women and their families attach special importance to nutrition during pregnancy. Most pregnant women eat finely, but they barely do any activities, resulting in excessive prevalence of nutritional excess during pregnancy, which increases the prevalence of pregnancy complications. Nowadays, an early diagnosis and treatment have become important measures to reduce maternal and fetal complications and improve quality ${ }^{[8]}$.If the blood glucose level is not timely and effectively controlled, pregnant women may suffer from miscarriage, excessive amniotic fluid, premature delivery, hypertension during pregnancy, infection, and perinatal infants may present with fetal distress, neonatal asphyxia, and even maternal and child deaths. It treatment is not timely adopted, a 
series of bad outcomes occur to mother and baby, such as dystocia, neonatal brachial plexus injury ${ }^{[9-10]}$. Recently, fetuses with gestational diabetes often suffer from limited growth and development, or a large child causes maternal dystocia or causes severe birth injury and increases complications such as cesarean section rate and suffocation. The morbidity and mortality rate show a significant upward trend which limit fetal growth and development, and will make the incidence of neonatal related diseases such as respiratory distress syndrome, polycythemia, etc., which may also bring a negative effect on maternal prognosis and fetal quality ${ }^{[11]}$. Therefore, it is a critical time to increase the obstetrician's attention to this issue. In our hospital reasonable interventions are adopted for pregnant women during pregnancy to improve the outcome of pregnancy.

In this study, after nutrition and health education as well as targeted nutritional guidance, the incidence of gestational diabetes, hypertension, macrosomia, and fetal distress in the nutrition group show a significant downward trend. Compared with the control group, the difference between groups is significant $(\mathrm{P}<0.05)$; The incidence of anemia after delivery in the nutritional group is significantly lower, and the incidence of maternal natural childbirth rate is significantly higher, with significant differences between the groups $(\mathrm{P}<0.05)$. This shows that the indicators of pregnancy during nutrition and health education as well as targeted nutrition interventions have been significantly improved, and the quality of patients' life has also been significantly improved. The adoption of health education and nutritional guidance for mothers can effectively improve the outcome of childbirth and can accelerate the birth time.

To sum up, through continuous exploration and clinical validation for pregnant patients, the implementation of selective, focused, staged, targeted nutrition and health education as well as nutrition guidance interventions can improve the index control and delivery outcomes of pregnant patients and at the same time effectively reduce the various complications of maternal and neonatal perinatal period. It shows superiority: increase the natural delivery rate, reduce the neonatal asphyxia rate and maternal and child complications, which will be more conducive to the maternal delivery process with a certain guiding value in clinical practice, and is worthy of clinical reference.

\section{References}

[1] Shi Liya. Significance of nutrition guidance and maternal and child health guidance in pregnant and perinatal period[J]. China Health Care Nutrition (Late edition), 2013, 23 (3) : 11791180.

[2] Niu Xiaohu, Zhang Guoying, Luo Xiaoming etc. Analysis of the factors influencing the nutrition of early and middle-term pregnant women and their behavior[J]. Maternal and Child Health Care of China, 2013, 28 (2) :268-271.

[3] Fu Yuye. Influence of perinatal nutrition health education on maternal health care [J]. The Chinese and foreign health abstract, 2014, 11 ( 19) : 117-118.

[4] Yu Yingjie. Analysis of perinatal nutrition health education on maternal health care[J]. Chinese Journal of Modern Drug Application, 2013, 7 ( 20) : 238-238.

[5] Xuan Wanru, Chen Jinhong, Wang Juxian etc. Analysis of the factors influencing the dietary behavior of pregnant women based on individualized nutrition health education[J]. Medicine and Society, 2013, 26 ( 11) :22-24.

[6] Chen Rujuan, Lin Ruiru, Zhang Qiongbi etc. Effect of perinatal nutrition health education on pregnant women's dietary behavior[J]. China Medicine and Pharmacy, 2015, 5 ( 14) : 66-68, 77.

[7] Zou Xiaoyan. Study on the improvement of nutritional status and reproductive outcome of pregnant women by zinc supplementation intervention[J]. Journal of clinical medical literature, 2015,2 ( 25) : 5181-5182.

[8] Liang Denghui, Li Mingliang, Song Yubao etc.Clinical effect of different nutrition intervention methods on nutrition guidance of pregnant women in perinatal period and follow-up observation of neonatal birth weight and health status[J]. Maternal and Child Health Care of China. 
[9] Wang Aicun.Effect of nutrition guidance during pregnancy on health care of perinatal pregnant women[J]. Healthy People, 2015, 21 (4) : 156-157.

[10] Sun Ming. Effect of dietary nutrition guidance during pregnancy on nutritional status and pregnancy outcome during pregnancy[J]. Chinese Journal of Public Health, 2015, 31 ( 3) : 367 $-368$.

[11] Zhou Yajuan, Xiayun, Chen Guihua etc. Investigation and analysis on nutrition knowledge and dietary habit of pregnant women[J]. Guizhou Medical Journal, 2014, 39 ( 12) : 1132-1133. 